\title{
Asymptomatic COVID-19: What the Neuroradiologist Needs to Know about Pulmonary Manifestations
}

\author{
(D) R.F. Barajas, Jr, (D) G. Rufener, (D). Starkey, (D)T. Duncan, and (D) C. Fuss
}

\begin{abstract}
SUMMARY: Coronavirus disease 2019 (COVID-19) is an infectious disease with a high asymptomatic incidence. Asymptomatic infections within a population will inevitably lead to diagnosis via unrelated medical imaging. We report the case of an asymptomatic patient undergoing a spine CT examination for trauma who was incidentally found to have lung abnormalities later confirmed to be COVID-19. We aim to familiarize neuroradiologists with the spectrum of COVID-19 pulmonary manifestations that are likely to be observed on neck and spine CT imaging.
\end{abstract}

ABBREVIATIONS: COVID-19 = coronavirus disease 2019; GGO = ground glass opacities; SARS-CoV-2 = Severe Acute Respiratory Syndrome-coronavirus 2

A novel coronavirus, Severe Acute Respiratory Syndrome-coronavirus 2 (SARS-CoV-2) or World Health Organization designated coronavirus disease 2019 (COVID-19) has developed into a pandemic. ${ }^{1,2}$ Initial reports suggest that COVID-19 is a highly infectious disease transmitted through respiratory droplets and fomites, resembling the spread of influenza. ${ }^{3}$ Unlike influenza, COVID-19 appears to have a prolonged incubation period with a median of 5.1 days but up to 14 days from exposure. ${ }^{4}$ The transmission rate is likely heightened by infected patients demonstrating little or no respiratory symptoms. The rate of asymptomatic infections remains unknown; however, data from the Diamond Princess Cruise Ship and Washington State Skilled Nursing Facility cohorts suggest that approximately 50\% of the patients with confirmed COVID-19 were asymptomatic at the time of diagnosis. ${ }^{4,5}$

Received April 4, 2020; accepted after revision April 9

From the Departments of Radiology (R.F.B., Jr, G.R., J.S., C.F.); Advanced Imaging Research Center (R.F.B., Jr); and Knight Cancer Institute, Translational Oncology Research Program (R.F.B., Jr), Oregon Health \& Science University, Portland, Oregon; and Departments of Radiology (T.D.), Portland Veterans Affairs Medical Center, Portland, Oregon.

This material is the result of work supported by resources and the use of facilities at the Portland Veterans Affairs Medical Center.

The content does not represent the views of the United States Department of Veterans Affairs or the United States Government.

This work was supported, in part, by National Institutes of Health grant KL2TR002370 (R.F.B., Jr).

Please address correspondence to Ramon F. Barajas, Jr, MD, Department of Radiology, Neuroradiology Section, Oregon Health \& Science University, L340 3181 S.W. Sam Jackson Park Rd., Portland, OR 97239; e-mail: barajaslab@ohsu.edu; @OHSURadiology

- Indicates open access to non-subscribers at www.ajnr.org

http://dx.doi.org/10.3174/ajnr.A6561
The potential of a highly infectious disease spreading via asymptomatic carriers poses unique challenges in the radiologic setting because risk stratification and isolation protocols depend on a suggestive clinical history. Likewise, the presence of a large, asymptomatic cohort will inevitably lead to incidental diagnoses. We report the case of an asymptomatic patient undergoing a cervical spine CT examination for trauma who was incidentally found to have lung abnormalities, later confirmed to be COVID19. Given the escalating incidence, the aim of this report is to familiarize neuroradiologists with the spectrum of COVID-19 pulmonary manifestations that are likely to be observed on neck and spine CT examinations.

\section{Brief Report}

An 83-year-old man with a history of chronic obstructive pulmonary disease and diabetes mellitus type 2 presented to our institution with 3 days of progressive altered mental status culminating in a fall from standing. A chronic infrequent dry cough was reported, but acute respiratory symptoms were denied. Initial physical examination and vital sign assessment were unremarkable. CT of the cervical spine for suspected traumatic injury showed incidental lung findings of peripheral bilateral apical ground glass opacities (GGO) with a crazy paving appearance (Figure). The reporting neuroradiologist (R.F.B., Jr) consulted the cardiothoracic radiology section chief (C.F.) regarding possible COVID-19 infection. It was agreed that the recently published Radiological Society of North America (RSNA) Expert Consensus Statement on Reporting Chest CT Findings Related to COVID-19 should be used. ${ }^{6}$ The treating emergency medicine physician was notified of the high suspicion for 


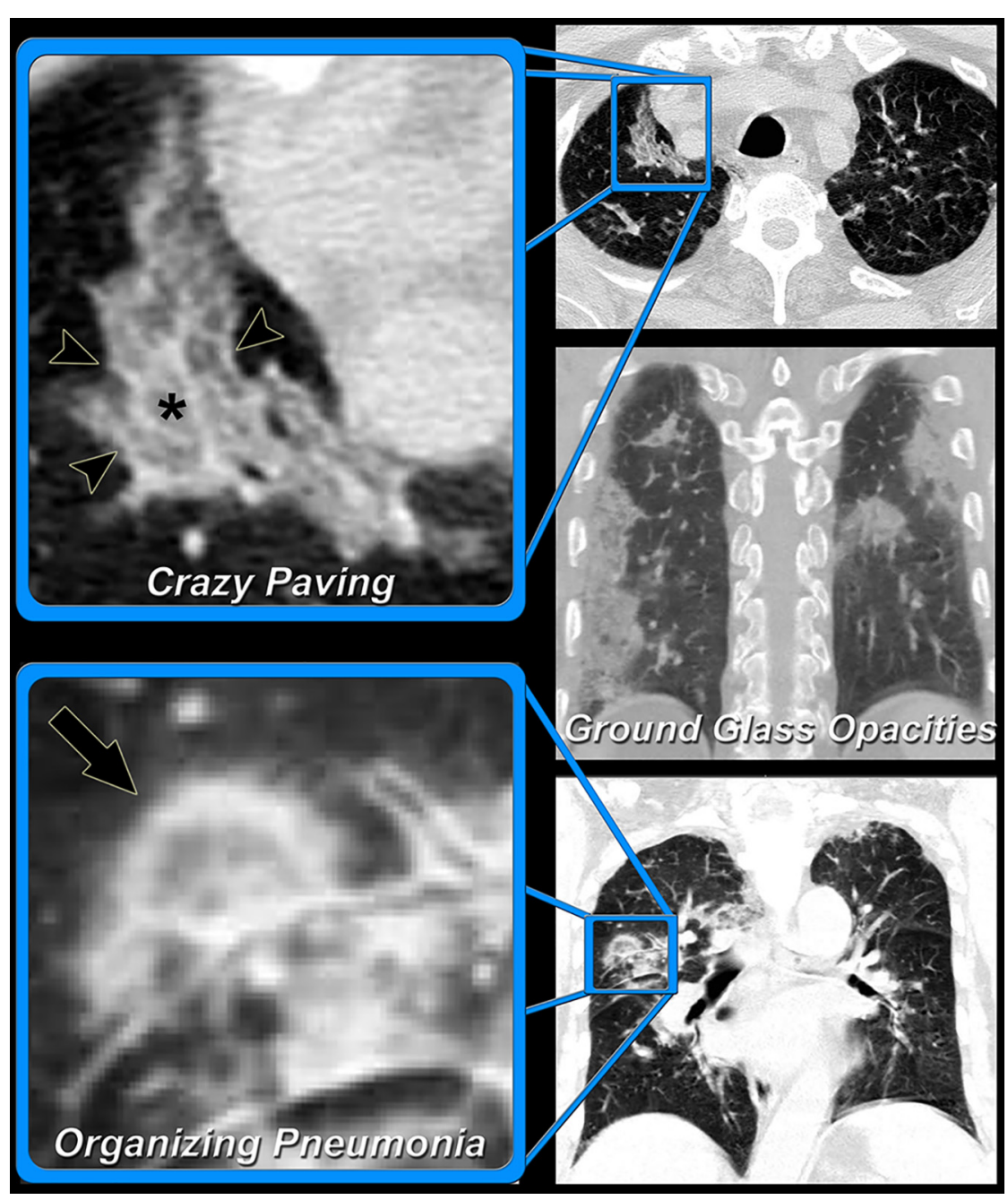

FIGURE. Pulmonary manifestations of asymptomatic COVID-19 Infection. An 83-year-old man without acute respiratory symptoms presented to our institution for $\mathrm{CT}$ of the cervical spine following a fall from standing. Limited evaluation of the lung apex on the cervical spine CT (upper row) demonstrated bilateral GGO (asterisk) with associated interlobular septal thickening (arrowheads, crazy paving), a typical appearance for COVID-19 infection. After consultation with the treating emergency department physician, the patient was placed on droplet precautions, a COVID-19 viral test was obtained, and CT of the chest was performed to assess the extent of disease. Bilateral peripheral GGO (middle row) and organizing pneumonia (arrow) were observed (lower row). The patient's GeneXpert COVID-19 test subsequently had positive findings, and the patient was admitted to the medical intensive care unit for observation. This case highlights the importance of familiarity with the pulmonary manifestations of COVID-19 and current expert consensus reporting recommendations. This familiarity will ensure timely, optimal patient care in the current pandemic setting.

\section{DISCUSSION}

Our case highlights the importance of the neuroradiologist being familiar with pulmonary CT findings associated with COVID-19. The neuroradiologist may be the first provider to recognize the possibility of a COVID-19 infection. This role places the responsibility of alerting the treating physician so that standard operating procedures for infection and exposure control can be initiated.

The RSNA Expert Consensus Statement on Reporting Chest CT Findings Related to COVID-19 has recently provided an up-to-date summary of published literature. ${ }^{6}$ Patients with symptomatic COVID-19 infections typically present with GGO with or without superimposed consolidations. Pulmonary consolidation has been reported as peripheral, posterior, and diffuse with a predominate lower lung zone distribution. The GGO can have a rounded morphology with superimposed inter- or intralobular septal thickening, termed "crazy paving." The GGO associated with COVID-19 do not seem to follow a perihilar pattern. Later stages of the disease can demonstrate an organizing pneumonia manifested by a "reversed halo sign" or "atoll sign." The aforementioned pulmonary CT findings are neither sensitive nor specific. Indeed, GGO and crazy paving are observed in many viral lung infections and inflammatory diseases.

The RSNA Expert Consensus Statement on Reporting Chest CT Findings Related to COVID-19 has provided structured language to assist radiologists in reducing reporting variability and uncertainty (Table). ${ }^{6}$ While
COVID-19 viral pneumonia, and standard protocols for infection and exposure control were initiated.

On the same day, a GeneXpert test (Cepheid) was reported positive for COVID-19 (SARS-CoV-2). The patient was admitted to the medical intensive care unit because of age and comorbidities. Findings of a standard viral panel were negative, including influenza A and B. Labs were notable for thrombocytopenia, mild leukopenia, and mildly elevated D-dimer levels. An experimental course of hydroxychloroquine was initiated. On hospital day 3 , the patient experienced a fever of $38.9^{\circ} \mathrm{C}$ with waxing and waning delirium. Nevertheless, the patient remained on room air without shortness of breath or progressive respiratory symptoms. The patient remains in the medical intensive care unit at the time of this writing. focused on the reporting of chest CT findings, it could also be useful to the neuroradiologist in describing incidentally observed pulmonary abnormalities. Four reporting categories based on pulmonary CT findings have been proposed, and reporting language is suggested. We propose, in the context of the ongoing pandemic, that the presence of typical and indeterminate pulmonary imaging findings on neck or spine CT examinations should prompt the neuroradiologist to discuss the possibility of COVID-19 infection with the treating physician in an expedited manner. Pulmonary manifestations of COVID-19 seem to be dependent on the patient's disease state at the time of imaging. A comprehensive study of pulmonary findings in asymptomatic patients with COVID-19 has not been reported. 


\begin{tabular}{|c|c|c|}
\hline $\begin{array}{l}\text { CT Imaging } \\
\text { Appearance }\end{array}$ & Pulmonary CT Findings & Suggested Reporting Language \\
\hline Typical & $\begin{array}{l}\text { Crazy paving } \\
\text { Bilateral } \\
\text { Peripheral } \\
\text { GGO } \\
\text { Bilateral } \\
\text { Peripheral } \\
\text { Multifocal } \\
\text { Rounded } \\
\text { Organizing pneumonia }\end{array}$ & $\begin{array}{l}\text { "Commonly reported imaging features of (COVID-19) pneumonia are present. } \\
\text { Other processes such as influenza pneumonia and organizing pneumonia, } \\
\text { as can be seen with drug toxicity and connective tissue disease, can cause a } \\
\text { similar imaging pattern." }\end{array}$ \\
\hline Indeterminate & $\begin{array}{l}\text { No typical features and } \\
\text { GGO } \\
\text { Multifocal } \\
\text { Diffuse } \\
\text { Perihilar } \\
\text { Unilateral } \\
\text { Nonrounded }\end{array}$ & $\begin{array}{l}\text { "Imaging features can be seen with (COVID-19) pneumonia, though are } \\
\text { nonspecific and can occur with a variety of infectious and noninfectious } \\
\text { processes." }\end{array}$ \\
\hline Atypical & $\begin{array}{l}\text { No typical or indeterminate features } \\
\text { and } \\
\text { Isolated lobar or segmental } \\
\text { consolidation without GGO } \\
\text { Centrilobular nodules } \\
\text { Cavitation } \\
\text { Smooth interlobular septal thickening } \\
\text { with pleural effusion }\end{array}$ & $\begin{array}{l}\text { "Imaging features are atypical or uncommonly reported for (COVID-19) } \\
\text { pneumonia. Alternative diagnosis should be considered." }\end{array}$ \\
\hline Negative & No CT features of pneumonia & $\begin{array}{l}\text { "No CT findings present to indicate pneumonia. (Note: CT may be negative in } \\
\text { the early stages of COVID-19.)" }\end{array}$ \\
\hline
\end{tabular}

${ }^{a}$ This table was adapted from Simpson et al. ${ }^{6}$ Crazy paving is rounded ground glass opacification associated with inter- and intralobular septal thickening. Organizing pneumonia is a focus of GGO with peripheral consolidation previously described as "atoll sign" or "reverse halo sign."

As of April 2020, the RSNA, Society of Thoracic Radiology, and the American College of Radiology (ACR) recommend against CT imaging being used to screen for or as a first-line test to diagnose COVID-19. ${ }^{6}$ The ACR highlights the limitations of using CT for COVID-19 diagnosis. Normal findings on chest CT do not mean that an individual does not have a COVID-19 infection. Likewise, abnormal CT findings are not sufficiently specific to establish the diagnosis.

In conclusion, ground glass opacities, crazy paving, and organizing pneumonia encountered on pulmonary CT scans should prompt consideration of COVID-19 infection in the current pandemic, with expedited communication with the referring providers and follow-up testing as appropriate.

\section{ACKNOWLEDGMENTS}

The first author thanks Bethany Barajas, MSN, for her helpful comments regarding this article, the clinical colleagues who are risking their health to treat patients with COVID-19, and the many patients who are confronting a deadly disease.

Disclosures: Ramon F. Barajas - RELATED: Grant. National Institutes of Health, Comments: National Institutes of Health Career Training Grant KL2TR002370.* Timothy Duncan — UNRELATED: Employment: Portland Veterans Affairs Medical Center, Comments: I am a VA staff physician. Cristina Fuss-OTHER RELATIONSHIPS: My husband is a shareholder at ViewRay Inc, which specializes in the Magnetic Resonance Imaging Guided Linear Accelerator. *Money paid to the institution.

\section{REFERENCES}

1. World Health Organization. Novel coronavirus (2019-nCoV) situation report-15. February 4, 2020. https://www.who.int/docs/ default-source/coronaviruse/situation-reports/20200204-sitrep-15-ncov. pdf?sfvrsn=88fe8ad6_2. Accessed April 3, 2020

2. World Health Organization. WHO Director-General's opening remarks at the media briefing on COVID-19. March 11, 2020. https:/www.who.int/dg/speeches/detail/who-director-general-s-openingremarks-at-the-media-briefing-on-covid-19-11-march-2020. Accessed April 3, 2020

3. Lauer SA, Grantz KH, Bi Q, et al. The incubation period of coronavirus disease 2019 (COVID-19) from publicly reported confirmed cases: estimation and application. Ann Intern Med 2020 Mar 10. [Epub ahead of print] CrossRef Medline

4. Japanese National Institute of Infectious Diseases. Field Briefing: Diamond Princess COVID-19 Cases. 20 Feb Update. https://www.niid.go.jp/niid/ en/2019-ncov-e/9417-covid-dp-fe-02.html. Accessed April 3, 2020

5. Kimball A, Hatfield KM, Arons $\mathrm{M}$, et al. Asymptomatic and Presymptomatic SARS-CoV-2 Infections in Residents of a Long-Term Care Skilled Nursing Facility-King County, Washington, March 2020. Morbidity and Mortality Weekly Report (MMWR). https://www. cdc.gov/mmwr/volumes/69/wr/mm6913e1.htm. Accessed April 3, 2020

6. Simpson S, Kay FU, Abbara S, et al. Radiological Society of North America Expert Consensus Statement on Reporting Chest CT Findings Related to COVID-19: Endorsed by the Society of Thoracic Radiology, the American College of Radiology, and RSNA. Radiology: Cardiothoracic Imaging 2020;2:e200152 CrossRef 